\title{
Propagation with time-dependent Hamiltonian
}

\author{
Gang Huang ${ }^{1}$ \\ ${ }^{1}$ Johannes Gutenberg University of Mainz
}

July 16, 2020

\begin{abstract}
In this note, we introduce one basic concept in nonlinear optical spectroscopy: time-dependent Hamiltonian. Then we give one example of application of the time evolution operator.
\end{abstract}

APS/123-QED

Institute for Physics, Johannes Gutenberg University, Mainz, Germany gang@uni-mainz.de

In optical spectroscopy, the choice we face is: (1) working with a time-independent Hamiltonian in a larger phase space that includes the matter and the radiation field(Shaul Mukamel, 1995); (2) using a timedependent Hamiltonian in a smaller phase space of the matter alone.

For any vector $|\psi\rangle$ in Hilbert space, its dynamical equation is the time-dependent Schrodinger equation:

$$
i \hbar \frac{\partial|\psi(t)\rangle}{\partial t}=\mathbf{H}|\psi(t)\rangle
$$

Since

$$
|\psi(t)\rangle=\sum_{l}\left|f_{l}\right\rangle\left\langle f_{l} \mid \psi(t)\right\rangle
$$

and

$$
\mathbf{H}\left|f_{l}\right\rangle=E_{l}\left|f_{l}\right\rangle,
$$

we have

$$
i \hbar \frac{\partial}{\partial t}\left\langle f_{l} \mid \psi(t)\right\rangle=E_{l}\left\langle f_{l} \mid \psi(t)\right\rangle,
$$

which is

$$
i \hbar \frac{\partial}{\partial t} c_{l}=E_{l} c_{l},
$$

or

$$
\mathbf{H c}=E \mathbf{c} .
$$

We obtain the wave function at time $t$ :

$$
\left\langle f_{l} \mid \psi(t)\right\rangle=e^{-\frac{i E_{l}\left(t-t_{0}\right)}{\hbar}}\left\langle f_{l} \mid \psi\left(t_{0}\right)\right\rangle,
$$


where $\left\langle f_{l} \mid \psi\left(t_{0}\right)\right\rangle$ is the initial expansion coefficients of the wavefunction. We then have

$$
|\psi(t)\rangle=\sum_{l} e^{-\frac{i E_{l}\left(t-t_{0}\right)}{\hbar}}\left|f_{l}\right\rangle\left\langle f_{l} \mid \psi\left(t_{0}\right)\right\rangle
$$

Therefore, the evolution operator $U\left(t, t_{0}\right)$ can be defined as:

$$
|\psi(t)\rangle \equiv U\left(t, t_{0}\right)\left|\psi\left(t_{0}\right)\right\rangle
$$

or

$$
U\left(t, t_{0}\right) \equiv \sum_{l}\left|f_{l}\right\rangle e^{-\frac{i E_{l}\left(t-t_{0}\right)}{\hbar}}\left\langle f_{l}\right|
$$

It is immediately follows that

$$
U\left(t_{0}, t_{0}\right) \mid=1
$$

The eq. 7 gives the evolution operator in a specific representation, i.e., the eigenstates of the Hamiltonian H.

Here is one example of application of the time evolution operator. Calculate the time evolution operator of a coupled 2-level system $\left(\left|\psi_{a}\right\rangle\right.$ and $\left.\left|\psi_{b}\right\rangle\right)$ with energies $\epsilon_{a}, \epsilon_{b}$, and a coupling $V_{a b}$, represented by the Hamiltonian

$$
\left[\begin{array}{cc}
\epsilon_{a} & V_{a b} \\
V_{b a} & \epsilon_{b}
\end{array}\right]
$$

Solution: Denote

$$
V_{a b}=V_{b a}^{*}=\left|V_{a b}\right| e^{-i \chi}(0<\chi<\pi / 2) .
$$

Denote $\lambda$ as the eigenvalue of the energy, solve the JiuQi equation

$$
\left(\epsilon_{a}-\lambda\right)\left(\epsilon_{b}-\lambda\right)-\left|V_{a b}\right|^{2}=0,
$$

we get the eigenvalue of the energy: $\lambda_{ \pm}=\frac{\left(\epsilon_{a}+\epsilon_{b}\right) \pm \sqrt{\left(\epsilon_{a}-\epsilon_{b}\right)^{2}+4\left|V_{a b}\right|^{2}}}{2}$. Then the eigenstates can be calculated. For $\lambda=\lambda_{-}$,

$$
\left(\epsilon_{b}-\lambda_{-}\right) b=-V_{a b} e^{i \chi} a,
$$


i.e.,

$$
\begin{gathered}
\frac{b}{a}=\frac{-\left|V_{a b}\right| e^{i \chi}}{\epsilon_{b}-\lambda} \\
=\frac{-2\left|V_{a b}\right| e^{i \chi}}{\left(\epsilon_{b}-\epsilon_{a}\right)+\sqrt{\left(\epsilon_{a}-\epsilon_{b}\right)^{2}+4\left|V_{a b}\right|^{2}}} \\
=\frac{-2\left|V_{a b}\right| e^{i \chi} /\left(\epsilon_{a}-\epsilon_{b}\right)}{-1+\sqrt{1+\frac{4\left|V_{a b}\right|^{2}}{\left(\epsilon_{a}-\epsilon_{b}\right)^{2}}}} \\
=\frac{-\tan 2 \theta}{-1+\sec 2 \theta} e^{i \chi} \\
=-\frac{\cos \theta}{\sin \theta} e^{i \chi},
\end{gathered}
$$

where we have set

$$
\tan 2 \theta \equiv \frac{2\left|V_{a b}\right|}{\epsilon_{a}-\epsilon_{b}}, 0<\theta<\frac{\pi}{2}
$$

Therefore,

$$
\left|\psi_{-}\right\rangle=\left[\begin{array}{c}
-\sin \theta e^{-i \chi / 2} \\
\cos \theta e^{i \chi / 2}
\end{array}\right] .
$$

Similarly, replace $\lambda_{-}$by $\lambda_{+}$, we can obtain

$$
\left|\psi_{+}\right\rangle=\left[\begin{array}{c}
\cos \theta e^{-i \chi / 2} \\
\sin \theta e^{i \chi / 2}
\end{array}\right]
$$

Thus,from eq. 7 , the time evolution operator is

$$
U\left(t, t_{0}\right)=\left|\psi_{+}\right\rangle\left\langle\psi_{+}\left|e^{-\frac{i}{\hbar} \lambda_{+}\left(t-t_{0}\right)}+\right| \psi_{-}\right\rangle\left\langle\psi_{-}\right| e^{-\frac{i}{\hbar} \lambda_{-}\left(t-t_{0}\right)} .
$$

Using eq.( 13) and (14), we obtain the exprssion of $U\left(t, t_{0}\right)$ :

$$
U\left(t, t_{0}\right)=
$$

$$
\begin{aligned}
& {\left[\begin{array}{cc}
\cos ^{2} \theta & \cos \theta \sin \theta e^{-i \chi} \\
\cos \theta \sin \theta e^{i \chi} & \sin ^{2} \theta
\end{array}\right] \mathrm{e}^{-\frac{i}{\hbar} \lambda_{+}\left(t-t_{0}\right)}+} \\
& + \\
& {\left[\begin{array}{cc}
\sin ^{2} \theta & -\cos \theta \sin \theta e^{-i \chi} \\
-\cos \theta \sin \theta e^{i \chi} & \cos ^{2} \theta
\end{array}\right] \mathrm{e}^{-\frac{i}{\hbar} \lambda_{-}\left(t-t_{0}\right)} .}
\end{aligned}
$$

Discussion: suppose the system is initially (at time $t_{0}=0$ ) in the $\left|\phi_{a}\right\rangle$ state, i.e., $|\psi(0)\rangle=\left|\phi_{a}\right\rangle$. We can calculate the probability of the system to be found in the $\left|\phi_{b}\right\rangle$ state at time $t$ 


$$
\begin{array}{cc}
P_{b a}(t) \quad & =\left|\left\langle\phi_{b} \mid \psi(t)\right\rangle\right|^{2} \\
= & \left|\left\langle\phi_{b} \mid U\left(t, t_{0}\right) \psi(0)\right\rangle\right|^{2} \\
= & \left|\left\langle\phi_{b}\left|U\left(t, t_{0}\right)\right| \phi_{a}\right\rangle\right|^{2}
\end{array}
$$

(18)

Since

$$
\left\langle\phi_{b}\left|U\left(t, t_{0}\right)\right| \phi_{a}\right\rangle=
$$

$\left[\begin{array}{ll}0 & 1\end{array}\right]$

$\left[\begin{array}{ll}U_{a a}(t) & U_{a b}(t) \\ U_{b a}(t) & U_{b b}(t)\end{array}\right]$

$\left[\begin{array}{ll}U_{b a}(t) & U_{b b}(t)\end{array}\right]$

$\left[\begin{array}{l}1 \\ 0\end{array}\right]$

$=\mathrm{U}_{b a}(t)$

$=\sin \theta \cos \theta e^{i \chi} e^{-\frac{i}{\hbar} \lambda_{+}\left(t-t_{0}\right)}-$

- $\sin \theta \cos \theta e^{i \chi} e^{-\frac{i}{\hbar} \lambda_{-}\left(t-t_{0}\right)}$

$=\sin 2 \theta e^{i \chi} \frac{\left(\cos \frac{\lambda_{+}\left(t-t_{0}\right)}{\hbar}-i \sin \frac{\lambda_{+}\left(t-t_{0}\right)}{\hbar}-\right.}{2}$

$-\cos \lambda_{-}\left(t-t_{0}\right) \frac{}{\left.\hbar+i \sin \frac{\lambda_{-}\left(t-t_{0}\right)}{\hbar}\right)}$

$=\sin 2 \theta e^{i \chi} \frac{}{2 \times 2 i \sin \beta(\cos \alpha-i \sin \alpha)}$

$=\operatorname{isin} 2 \theta e^{i(\chi-\alpha)} \sin \beta,(13)$ where we have defined

$$
\alpha=\frac{\left(\epsilon_{a}+\epsilon_{b}\right)\left(t-t_{0}\right)}{2 \hbar}, \beta=\frac{\sqrt{\left(\epsilon_{a}-\epsilon_{b}\right)^{2}+4\left|V_{a b}\right|^{2}}\left(t-t_{0}\right)}{2 \hbar} .
$$

So

$$
\begin{gathered}
\quad=\sin ^{2} 2 \theta \sin ^{2} \beta \\
=\frac{\left.4\left|\phi_{b}\right| U\left(t, t_{0}\right)\left|\phi_{a}\right\rangle\right|^{2}}{\sqrt{\left(\epsilon_{a}-\epsilon_{b}\right)^{2}+4\left|V_{a b}\right|^{2}}} \sin ^{2} \frac{\sqrt{\left(\epsilon_{a}-\epsilon_{b}\right)^{2}+4\left|V_{a b}\right|^{2}}\left(t-t_{0}\right)}{2 \hbar} .
\end{gathered}
$$

This is known as Rabi formula and

$$
\Omega_{R} \equiv \frac{\sqrt{\left(\epsilon_{a}-\epsilon_{b}\right)^{2}+4\left|V_{a b}\right|^{2}}}{\hbar}
$$


is known as Rabi frequency. For example, in the case of alkali atoms, the order of magnitude of the Rabi frequency is MHz. We assume that $\left(\epsilon_{a}-\epsilon_{b}\right)^{2}$ and $4\left|V_{a b}\right|^{2}$ have the same order of magnitude, i.e., $\frac{4\left|V_{a b}\right|^{2}}{\sqrt{\left(\epsilon_{a}-\epsilon_{b}\right)^{2}+4\left|V_{a b}\right|^{2}}} \sim \sqrt{\left(\epsilon_{a}-\epsilon_{b}\right)^{2}+4\left|V_{a b}\right|^{2}} \approx 10^{6}$.

\section{References}

(1995). 